\title{
Optimal Configuration of Multi-type Energy Storage for Integrated Energy System Considering Multi-energy Trading With Load Substitution
}

\author{
Jun Wang ${ }^{1, *}$, Wei Du ${ }^{1}$, Dongmei Yang ${ }^{1}$, Guoxin $\mathrm{He}^{1}$, and Xiaochen Zhang ${ }^{1}$ \\ ${ }^{1}$ NARI Technology Co., Ltd., Nanjing 210032, Jiangsu Province, China
}

\begin{abstract}
How to consider the impact of load substitution on user-side participating in multi-energy trading on system operation when configuring multi-type energy storage (MES) is an urgent problem that needs to be solved to improve the economics and energy efficiency of the integrated energy systems (IES). This paper presents an optimal configuration method of MES considering multi-energy trading with load substitution based on the characteristics of different energy supply seasons. Firstly, the multi-energy trading framework and MES configuration principle are proposed based on the structure of the IES. Secondly, a MES optimal configuration model for IES considering multi-energy trading with load substitution is established, then the IES trading strategies and MES planning schemes are solved. Finally, the proposed method is verified by an example. The results show that the proposed method reduces the MES configuration cost by $8.9 \%$. It can be seen that the MES configuration method proposed in this paper is helpful to repair the limitations of energy storage configuration research in IES and improve the economic benefits of the system.
\end{abstract}

\section{Introduction}

With the energy crisis and environmental pollution becoming more and more serious, the traditional fossil energy is increasingly depleted, and improving the efficiency of energy use, developing new energy sources and strengthening the comprehensive utilization of renewable energy are the inevitable choices for energy development. Integrated energy systems (IESs) can achieve energy interconnection, which is an important way to mitigate the energy crisis [1]. Energy storage systems as a key component of IES [2], with the ability to suppress load fluctuation to enhance renewable energy capacity, improve utilization of equipment, reduce energy costs [3]. However, with the diversification of user demand [4], the user-side integrated demand response resources are becoming more diverse. Load substitution can reflect the strong coupling relationship between electricity, gas and heat on the user side. Users can choose to use different energy sources to meet the same load demand, and when users change the demand for one or more energy sources, the demand for other energy sources will change accordingly. In addition, the differentiated production mode, supply system and consumption mode among different types of energy in the IES make the trading strategy and operation mode of multi-type energy storage (MES) including electric energy storage (EES), gas energy storage (GES) and heat energy storage (HES) more complex [5]. Therefore, it is an urgent problem that how IESs consider the multi-energy trading with loadsubstituting to configure the MES to improve the system economy and energy efficiency.

At present, a lot of research has been carried out on the optimal configuration of MES and the trading and operation of user side resources. In terms of configuration of MES, most of the existing researches are based on different benefit objectives to determine the equipment selection, equipment capacity and operation strategy of the system [6]. Reference [7] studied the optimal allocation of MES in regional IES considering multienergy complementarity, but only discussed the economy and feasibility of HES configuration. Reference [8] established an optimal operation model of microgrid IES including MES, but only the configuration of MES capacity is considered, the selection of MES is not involved. An optimal allocation model of electricity-heat integrated energy storage in cogeneration microgrid including P2G was constructed in [9] aiming at the multiobjective such as economy, reliability and new energy consumption rate. It can be seen that most of the existing configuration objects focus on the energy system with electric and heat load. The optimal configuration of energy storage is carried out considering the uncertainty, economy and feasibility. The consideration of the diversity of MES selection and the trading strategy of system operators is lacking. In terms of the trading and operation with user side resources, reference [10] put forward the optimal trading strategy for the integrated energy suppliers including renewable energy to stabilize the fluctuation through demand side means. Reference [11-12] proposed targeted trading strategies for non-cooperative trading and joint trading among multi-agent markets. Reference [13] 
proposed an optimal market trading strategy of load aggregators based on comprehensive demand response considering market information and demand side resource scheduling potential. It can be seen that most of the existing studies on the trading and operation based on the market mechanism and the characteristics of load demand response considering the diversity of operation modes of energy conversion equipment in energy hubs. Few studies have considered the impact of demand side multi energy load substitution on the IES, especially the mutual substitution relationship between gas load and electric load in the system.

In view of the existing research problems above, this paper studies the optimal configuration method of MES for IES considering multi-energy trading with load substitution. The main innovations of this paper are as follows:

1) For the configuration of the MES, we consider load substitution to participate in multi-energy trading of IES operators, and put forward the optimization selection method of the MES considering the load substitution ability of user-side resources and the multi-energy trading strategy of system operators. It is beneficial to enhance the economy of the IES.

2) Based on the MES optimal configuration method of IES considering multi-energy trading with load substitution, the influence of user load and energy price on the MES configuration results is analysed.

The main structure of this paper is as follows, Section 2 constructs a multi-energy trading framework for IES, and expounds the principle of MES configuration. Section 3 establishes a MES optimal configuration model for IES considering load substitution to participate in multienergy trading. Section 4 gives the solution flow of the model. Section $V$ analyses the effectiveness of the proposed method by example, and Section 5 summarizes the main findings of this paper.

\section{Multi-energy trading framework and principle of MES configuration for IES}

\subsection{Basic structure of IES}

The structure of IES has been elaborated in many literatures. Based on [14-15], the structure of IES in this paper is shown in Fig. 1. The IES include wind turbines (WT), combined heat and power (CHP), gas turbine (GT), power to gas (P2G), gas boiler (GB), electric boiler (EB), EES, GES and HES. All kinds of equipment supply, convert and store energy through electric supply bus (ESB), gas supply bus (GSB) and heat supply bus (HSB). In addition to supplying electrical, gas and heat load, it can also participate in energy trading through external energy supply center.

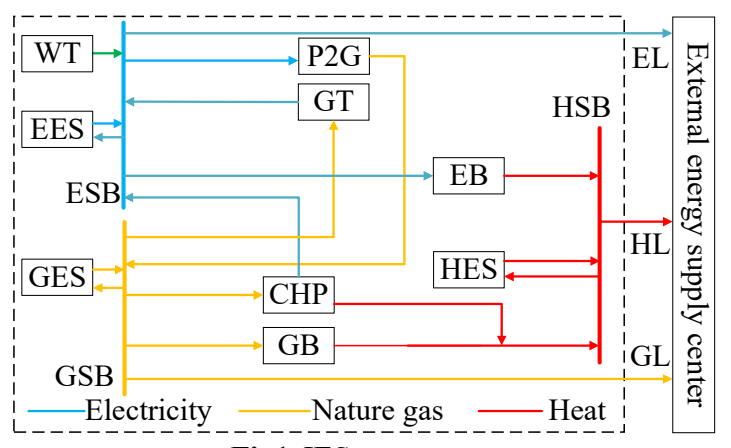

Fig1. IES structure.

\subsection{Load substitution}

Load substitution is reflected in the strong coupling between electricity, gas and heat. Users can choose to use different energy sources to meet the same load demand. When users change their demand for one or more energy sources, the demand for other energy sources may change [16]. This section considers the coupling between multienergy sources and establishes the gas-electric and heatelectrical load substitution models, as shown in formulas (1) and (2):

$$
\begin{aligned}
& \Delta P_{\mathrm{eg}, t}=\varepsilon_{\mathrm{eg}, t} P_{\mathrm{eg}, t}(1) \\
& \Delta P_{\mathrm{eh}, t}=\varepsilon_{\mathrm{eh}, t} P_{\mathrm{eh}, t}(2)
\end{aligned}
$$

Where, $\Delta P_{\text {eg, }, t}$ and $\Delta P_{\text {eh, }, t}$ are the changes of gas load and heat load produced by energy substitution in $t$ period. $\varepsilon_{\mathrm{eg}, t}$ and $\varepsilon_{\mathrm{eh}, t}$ are electricity-gas and electricity-heat substitution coefficients in $t$ period. $P_{\mathrm{eg}, t}$ and $P_{\mathrm{eh}, t}$ are the electricity load for substitution to gas and heat in $t$ period.

\subsection{Multi-energy trading framework}

Based on the existing research, the IES trading framework is constructed as shown in Fig. 2. The system operators of the IES participate in the trading of the multi-energy market, and flexibly transfer the load substitute resources on the user side through the price signals of electric, natural gas and heat energy.

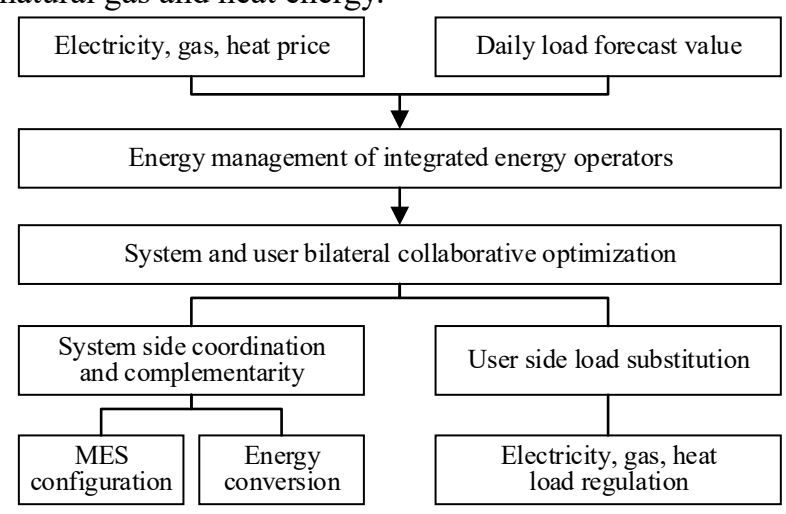

Fig2. IES trading framework.

According to the price signals of different energy markets, the following trading strategies are designed:

1) IES operators participate in the market for optimal decision-making considering load substitution of user-side resources.

2) Assuming that electricity and natural gas in IES can be sold to the outside, heat energy can only be consumed 
internally.

3) Electricity trading mode: the electricity load is supplied by the WT first, if insufficient, the natural gas purchased can be converted into electricity energy through the CHP, or the electric energy purchased from the market or discharged through the EES. If there is electricity surplus, sell electricity, or to recharge the EES, or to supply $\mathrm{P} 2 \mathrm{G}$ to convert to natural gas, or to supply EB to convert to heat.

4) Natural gas trading: to meet the demand of gas load, it may be purchased from the market, supplied by P2G or GES. The gas can be sold in excess of time, either to inflate the GES or to convert electricity and heat by CHP, or to convert heat through GB.

5) Heat trading: purchased from the market according to demand. Either use CHP to convert natural gas into heat energy, or through EB to convert electricity into heat energy to meet the heat load.

\subsection{Principle of MES configuration}

The principle of MES configuration in the IES in this paper is shown in Fig. 3. Because the system has different load characteristics in different seasons, the load curve and trading price of multi-energy is different in different seasons. Therefore, it is necessary to divide the scenarios to consider the characteristics of different seasons to optimize the configuration of MES. In this paper, four typical load scenarios are divided according to the seasons to form four typical daily load curves for the electrical, gas and heat loads in the system. The configuration period is the sum of four typical days multiplied by the number of days of the corresponding season. The configuration objects are EES, GES and HES. The multi-energy trading with load substitution of the system operator in section 2.3 is considered in the configuration.

\begin{tabular}{|c|c|}
\hline & Energy supply mode \\
Electrice & Natural gas Heat \\
\hline Planning options & Muli energy market \\
\hline LES \\
Substitution
\end{tabular}

Fig3. Principle of MES configuration.

\section{Optimal configuration model of MES for IES considering multi-energy trading with load substitution}

\subsection{Objective function}

The optimal configuration model of MES aims at the minimum total investment operating cost of the IES. The objective functions include equipment investment cost, operation and maintenance cost, and multi-energy trading cost, as shown in formula (3).

$$
\min C=C_{\text {inf }}+C_{\text {om }}+C_{\text {on }}
$$

Where, $C$ is the total investment and operation cost of the system. $C_{\text {inf }}$ is the equipment investment cost. $C_{\text {om }}$ is the operation and maintenance cost. $C_{\mathrm{on}}$ is the multienergy trading cost.

The cost of equipment investment is shown in formula (4) and (5).

$$
\begin{gathered}
C_{\text {inf }}=\sum_{m} \sum_{s \in S} \frac{C_{s, m} I_{s, m}}{365 T} D_{m} R_{s} \\
R_{s}=\frac{r(1+r)^{N_{s}}}{(1+r)^{N_{s}}-1}
\end{gathered}
$$

Where, $C_{s, m}$ is the construction cost of $s^{\text {th }}$ equipment to be selected. $s$ is the type number, indicating different capacity levels of the same equipment. $S$ is the alternative set of the construction capacity of the equipment to be selected. $I_{s, m}$ is the 0-1 decision variable, indicating whether the equipment is invested or not. $D_{m}$ is the number of days in the $m^{\text {th }}$ typical day. $T$ is the number of typical day intervals. $R_{S}$ is the investment recovery coefficient of $s^{\text {th }}$ equipment. $N_{s}$ is the service life of equipment. $r$ is the discount rate of $6.7 \%$.

The operating and maintenance costs is shown in formula (6).

$$
\begin{aligned}
C_{\mathrm{om}} & =\sum_{m} \sum_{t=1}^{T}\left[\sum_{x \in X} c_{x} P_{x}(m, t)\right. \\
& \left.+\sum_{s \in S} c_{s}\left|P_{\mathrm{sc}, s, t}(m, t)-P_{\mathrm{sd}, s, t}(m, t)\right|\right]
\end{aligned}
$$

Where, $T$ is the interval number in a typical day. $c_{x}$ is the unit power maintenance cost of built equipment. $P_{x}$ is the output of existing equipment $x$ in $m$ scenario in $t$ period. $c_{s}$ is the unit power maintenance cost of the energy storage equipment $s$ to be built. $P_{x}$ is the output of the existing equipment $x$ in the $m$ scenario in $t$ period. $P_{\mathrm{sc}, s, t}(m, t)$ and $P_{\mathrm{sd}, s, t}(m, t)$ represent the charging and discharging power of class $s$ energy storage devices in $t$ period under scenario $m$.

Multi-energy trading costs include the power cost of IES participating in the multi-energy market purchased from outside the system, as well as the heat and gas costs purchased, as shown in formula (7).

$$
C_{\mathrm{on}}=\sum_{m}\left[D_{m} \sum_{t=1}^{24} \sum_{y} c_{y, t} P_{m, y, t} \varphi_{m, y}\right]
$$

Where, $y$ is the type of energy. $c_{y, t}$ is the purchase price of energy $y$ in period $t . P_{m, y, t}$ is the purchase amount of energy $y$ in period $t$. The positive value is the purchase and the negative value is the sale. $\varphi_{m, y}$ is the energy price adjustment coefficient of energy $y$ in $m$ scenario.

\subsection{Constraints}

The constraints include power balance constraints of electrical, natural gas and heat, multi-energy trading constraints, MES operating constraints, CHP operating constraints, GB operating constraints, GT operating constraints, $\mathrm{P} 2 \mathrm{G}$ operating constraints, and EB operating constraints. The specific model expression can be found in [17-18], which will not be repeated here. 


\subsection{Solving process}

According to the MES optimal configuration model established in section 3.1 and 3.2, it can be seen that the optimization problem in this paper is a mixed integer nonlinear programming problem. Therefore, this paper takes the GAMS optimization software as the computing platform and calls the DICOPT solver to solve the problem. The specific solution process is as follows: first step, input wind power, load data and equipment parameters, energy price and other basic data. Considering the different energy supply season load and energy price characteristics, the specific application scenarios of IES planning are classified. The second step, for the energy trading problem of each scenario, consider the potential of user-side resources to participate in the trading and operation, and determine the optimal trading strategy. The third step, the multi-energy trading with load substitution participation is taken into account in the MES optimal configuration model of IES. The DICOPT solver is used to solve the problem on the GAMS platform, so as to determine the configuration scheme of MES and the optimal economic scheduling scheme. The solution flow is shown in Fig. 4.

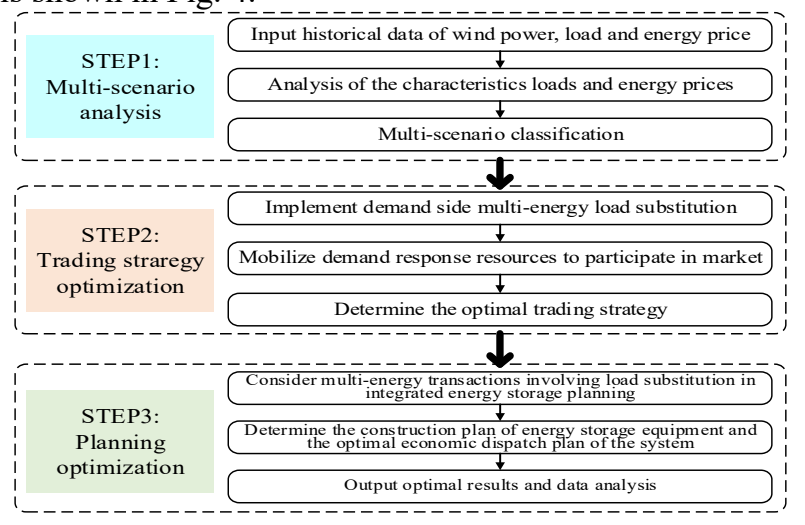

Fig4. Solution flow.

\section{Simulation results and analysis}

\subsection{Basic data}

This paper is based on the GAMS platforms on a Win10 operating system, i7CPU, $2.20 \mathrm{GHz}$ processor environment for the simulation and optimization analysis. To reduce the computational complexity of the model, the planning interval is reduced to four typical days. The investment cost takes into account the energy storage cost to be built, and it is amortized in order to balance its impact on simulation. The operating cost not only considers the running cost of energy storage, but also considers the operation and maintenance cost of other equipment, and the unit operating cost of the same equipment is the same in each scenario. The architecture of the example is shown in Fig. 1. Suppose the user side contains three types of energy load: electricity, gas and heat. The system has been equipped with WT, GB, EB, $\mathrm{CHP}$ and $\mathrm{P} 2 \mathrm{G}$. The equipment to be invested is three kinds of energy storage devices: EES, GES and HES. The load adjustment coefficient and the corresponding price adjustment coefficient under different scenarios are taken from [19]. The corresponding load curves, WT output, electricity and gas energy prices are referenced from [20]. The equipment types and parameters to be selected for MES are shown in Table 1 .

Table1. Parameters of candidate MES

\begin{tabular}{|c|c|c|c|c|}
\hline \multicolumn{2}{|r|}{ Equipment } & EES & GES & HES \\
\hline \multirow{3}{*}{ Type 1} & rated capacity (MW) & 1 & 0.8 & 0.8 \\
\hline & $\begin{array}{l}\text { Investment costs } \\
\text { (yuan } / \mathrm{KW} \cdot \mathrm{h} \text { ) }\end{array}$ & 600 & 130 & 50 \\
\hline & $\begin{array}{l}\text { Unit maintenance costs } \\
(\text { yuan } / \mathrm{KW} \cdot \mathrm{h})\end{array}$ & 0.018 & 0.017 & 0.016 \\
\hline \multirow{3}{*}{ Type2 } & rated capacity (MW) & 3 & 1 & 1 \\
\hline & $\begin{array}{l}\text { Investment costs } \\
\text { (yuan } / \mathrm{KW} \cdot \mathrm{h})\end{array}$ & 600 & 130 & 50 \\
\hline & $\begin{array}{l}\text { Unit maintenance costs } \\
\text { (yuan } / \mathrm{KW} \cdot \mathrm{h})\end{array}$ & 0.018 & 0.017 & 0.016 \\
\hline
\end{tabular}

\subsection{Effectiveness analysis of results}

\subsubsection{Analysis of investment construction costs}

In order to verify the effectiveness of the MES allocation method proposed in this paper in terms of investment construction cost, the following two situations are set up to compare:

S1: MES configuration for IES considering multienergy trading without load substitution

S2: MES configuration for IES considering multienergy trading with load substitution.

The MES configuration in two situations is shown in Table 2.

Table2. Scheme of MES configuration.

\begin{tabular}{|c|c|c|c|}
\hline \multicolumn{2}{|c|}{ MES } & $\begin{array}{c}\text { Capacity in } \\
\text { S1 }\end{array}$ & $\begin{array}{c}\text { Capacity in } \\
\text { S2 }\end{array}$ \\
\hline \multirow{2}{*}{ EES } & Type1 & 1 & 1 \\
\cline { 2 - 4 } & Type2 & - & - \\
\hline \multirow{2}{*}{ GES } & Type1 & 0.8 & 0.8 \\
\cline { 2 - 4 } & Type2 & - & - \\
\hline \multirow{2}{*}{ HES } & Type1 & 0.8 & 0.8 \\
\cline { 2 - 4 } & Type2 & - & 1 \\
\hline \multicolumn{2}{|c|}{$\begin{array}{c}\text { Investment construction } \\
\text { costs (104 yuan) }\end{array}$} & 10.25 & 10.72 \\
\hline \multicolumn{2}{|c|}{ Total system cost (10 $10^{4}$ yuan) } & 1436.5 & 1307.3 \\
\hline
\end{tabular}

From Table 2, we can see that S2 compared to S1, the EES and GES configuration is unchanged, but the HES configuration is increased. At this time, the overall energy storage configuration cost is increased, but the increase is small, only $4.38 \%$ is increased, and the total system cost is reduced by $8.9 \%$. mainly because the multi-energy trading in S2 considers load substitution, and the demand for electricity, gas and heat loads changes, which in turn affects the configuration and operation scheme of MES. It can be seen that considering the multi-energy trading with load substitution has an impact on the construction cost of MES, which can change the investment and construction of MES equipment. 


\subsubsection{Analysis of operating costs}

For verify the effectiveness of the MES configuration method proposed in this paper in terms of system operating cost, S3 is added on the basis of S1 and S2, specifically, neither load substitution nor investment in energy storage equipment is implemented. Cost of operation in three situations are $1426.23 \times 10^{4}$ yuan, $1296.55 \times 10^{4}$ yuan and $1447.01 \times 10^{4}$ yuan.

Compared with non-investment energy storage equipment, the total operating cost of the system that invest MES considering multi-energy trading without load substitution is reduced by $1.4 \%$, the total operating cost of the system that invest MES considering multi-energy trading with load substitution is reduced by $9.09 \%$. Therefore, the MES configuration and load substitution can play the advantage of multi-energy complementary and reduce the total operating cost of the system. However, the MES configuration considering multi-energy trading with load substitution is more able to improve the economic efficiency of the IES.

\subsection{Sensitivity analysis of results}

\subsubsection{Effect of load substitution on MES operation mode}

The operation of MES under different conditions in typical winter days is shown in Fig. 5.

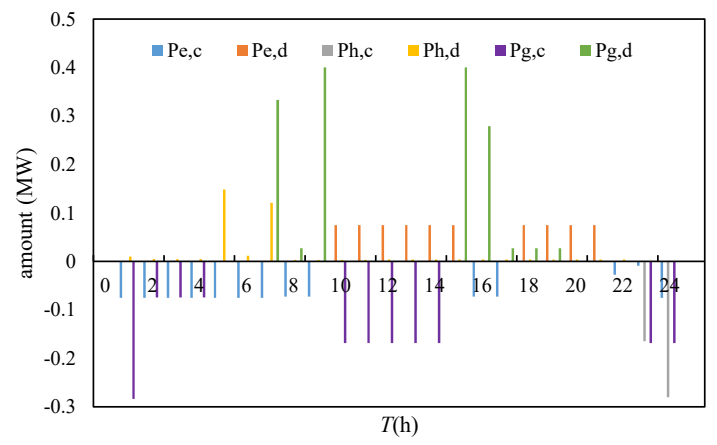

(a) MES operation under S1.

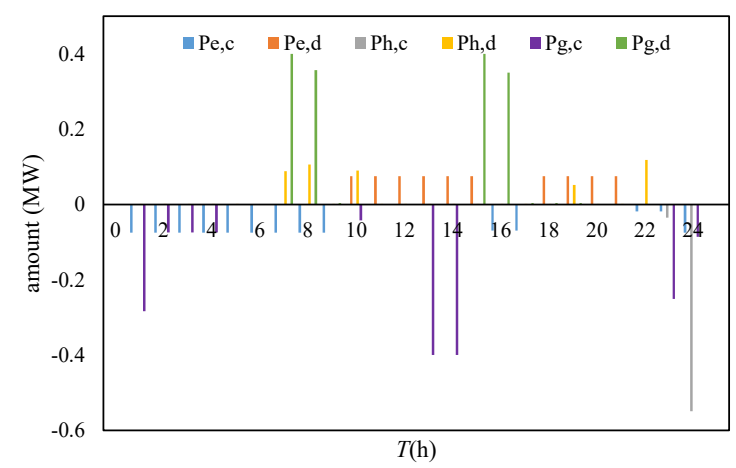

(b) MES operation under S2.

Fig5. MES operation under different situations.

It can be seen from Fig. 6 that after the load substitution participating the multi-energy trading, to meet the increased demand of heat load and gas load to implement electric-heat load substitution and electric-gas load substitution, the output force of EES changes in the flat period of electricity price, but the overall storage capacity of EES does not change. However, the total demand for the HES unit increased obviously, especially at the load valley period of 23:00 24:00, and the output of the HES unit increased significantly during the period, which increased by about $24 \%$. Compared with EES and HES devices, GES devices are more frequently charged and discharged, and the energy storage period is more frequent. It can be seen that after considering the multienergy trading with load substitution, the storage quantity of MES equipment is mainly optimized according to the change of the corresponding load under the premise of satisfying the overall demand of the multi-energy load.

\subsubsection{Effects of different periods of typical day on multi-energy trading strategies}

Because of the different load structure, the equipment output force of the typical day of different scenarios is different, and the corresponding trading strategy will be different. The specific analysis is carried out on the typical day of winter under S2. The trading strategies of electricity, natural gas and heat energy are shown in Fig. 6, Fig. 7 and Fig. 8.

As can be seen from Fig. 6, between 0:00 and 07:00, The main sources of electricity are market purchases and WT power. At this time the electricity load and prices are at a low point. The EES is charging, and $\mathrm{P} 2 \mathrm{G}$ and EB are running at full power. Thereafter, as the electricity load and price increase, between 10:00-15:00 and 18:00-21:00, because of the low WT output, far from meeting the electricity load, EES and CHP equipment began to release electricity. At this point, as the electricity price is at its peak, the electricity load is partially replaced by gas load and heat load, the actual peak electricity load decreases after substitution, approximately $1.24 \mathrm{MW}$, decreased by about $34.3 \%$. After $24: 00$, it is into the low period of electricity prices, electricity replacement is more economical, electricity load has increased. Therefore, through load substitution, it can be clearly seen that the demand for electricity load increases during the low period of electricity price, and the demand curve of electricity load decreases during the peak period of electricity price, which has the effect of cutting peak and filling valley.

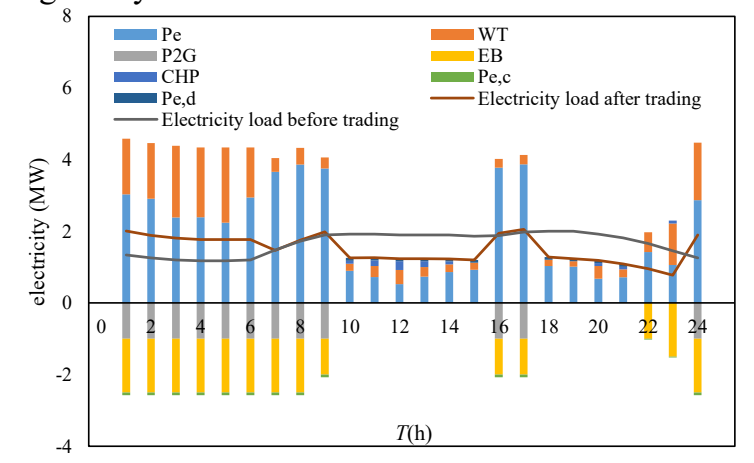

Fig6. Electricity trading.

It can be seen from Fig. 7 and Fig. 8 that the price of natural gas and heat energy is generally lower than the price of electricity, so we choose to buy more natural gas and heat energy. As an example, it can be seen that when 
the price difference is large, it is more economical to buy part of the heat energy from the market, while the other insufficient parts use the purchased natural gas to convert to heat energy through GB and CHP units. The EB heating is the main source of heat energy together. Through load substitution, the overall demand for heat energy and natural gas has increased to a certain extent, and the total demand has increased by $4.8 \%$ and $10.8 \%$ respectively. Of course, the corresponding heat energy and natural gas costs have also increased. However, despite the rising cost of natural gas and heat energy purchases due to load substitution, the scheme is still economically superior to the MES configuration of IES that do not take load substitution into account for multi-energy trading. The overall trading costs saved about $9.5 \%$. Therefore, the multi-energy trading strategy should be combined with the price of multi-energy markets to optimize the choice. Energy trading is not only affected by the energy price, the price of other energy will cross affect the supply of this energy.
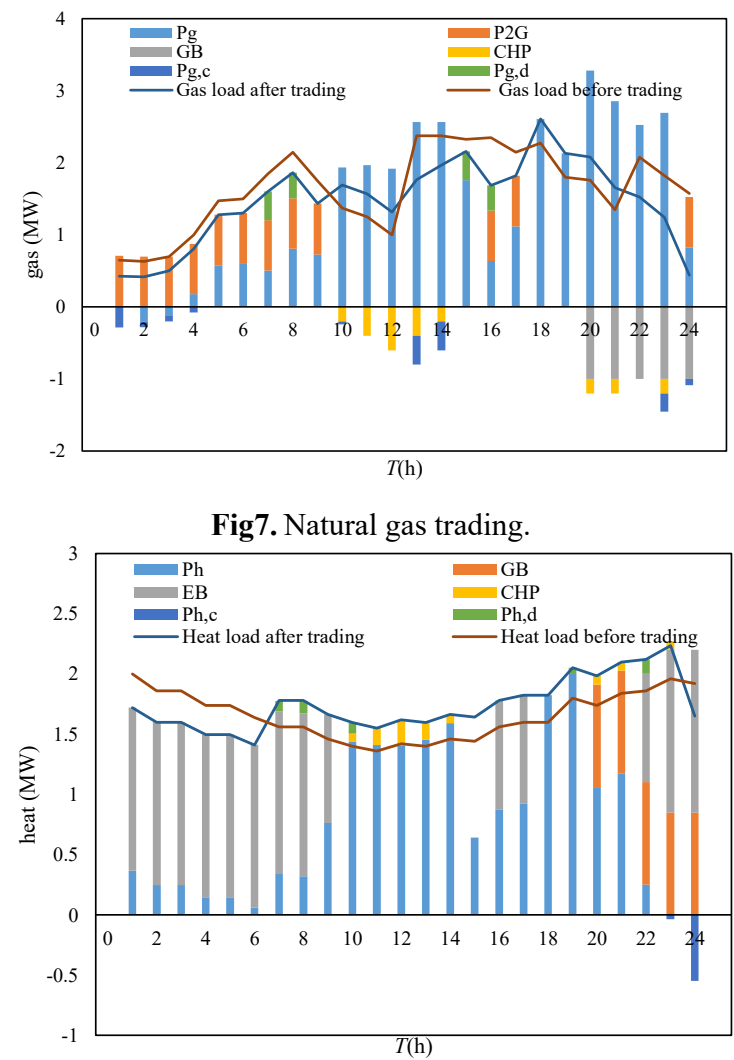

Fig8. Heat trading.

\section{Conclusions}

Aiming at the selection and allocation of the MES in IES, this paper proposes a MES optimal configuration method considering multi-energy trading with load substitution. The MES is configured to meet the energy supply and demand balance of different periods from different seasons. The following conclusions are obtained by examples:

(1) Load substitution participation in multi-energy trading can reduce the configuration cost of MES, which is $8.9 \%$ lower than that without load substitution.

(2) The characteristics of energy prices and loads affect the multi-energy load structure, the operation mode, and response capacity of EES, GES and HES.

(3) The IES network constraints will be taken into account to distinguish electricity, gas and heat trading to analyse the impact of network operating parameters on multi-energy trading at various times in the future work.

\section{Acknowledgments}

This work was supported in part by the Cooperative Research on Regional Integrated Energy Modeling Simulation and Operation Control Technology (No.BZ2019057).

\section{References}

1. H. J. Jia, Y. F. Mu, Y. Qi, INT J ELEC POWER, 54, 516 (2014)

2. C. Unishuay-Vila, J. W. Marangon-Lima, A. C. De Souza et al., IEEE Trans. Power Syst., 25, 1154 (2018)

3. Y. L. Xu, W. C. Wu, J. G. Zhou, IEEE Trans Smart Grid, 11, 686 (2020)

4. E. S. Parizy, H. R. Bahrami, S. S. Choi, IEEE Trans Smart Grid, 10, 3259 (2019)

5. G. Q. Li, R. F. Zhang, T. Jiang et al., Appl. Energy, 192, 408 (2017)

6. J. Qiu, Z. Y. Dong, J. H. Zhao et al. IEEE Trans. Power Syst., 30, 2119 (2015)

7. Y. H. Dai, Q. Chen, J. H. Hao et al. IEEE Trans Sustain Energy, 9, 1234 (2018)

8. C. Q. Ju, P. Wang, L. Goel et al. IEEE Trans Smart Grid, 9, 6047 (2018)

9. P. Rodrigo, B. Carlos, L. Fernando et al. IEEE Trans Smart Grid, 4, 996 (2013)

10. B. P. Hayes, A. Wilson, R. Webster et al. IET GENER TRANSM DIS, 10, 832 (2016)

11. C. Wang, Z. Y. Wang, Y. H. Hou et al. IEEE Trans. Power Syst., 33, 4958 (2018)

12. J. T. Chen, Q. Y. Zhu, IEEE Trans Smart Grid, 9, 6554 (2018)

13. D. Wang, Q. E. Hu, H. J. Jia et al. Appl. Energy, 248, 656 (2019)

14. C. C. Shao, M. Shahidehpour, X. F. Wang et al. IEEE Trans. Power Syst., 32, 4418 (2017)

15. C. B. Li, X. B. Liu, W. Zhang et al. IEEE Trans Smart Grid, 7, 481 (2016)

16. P. Y. Liu, T. Ding, Z. X. Zou et al. Appl. energy, 250, 512 (2019)

17. X. Dou, J. Wang, Z. Wang et al., J. Mod. Power Syst. Clean Energy, 8, 841 (2020)

18. X. Dou, J. Wang, Q. R. Hu et al., CSEE J POWER ENERGY (to be published)

19. A. Shahmohammadi, D. M. Moradi, H. Ghasemih et al. IEEE Trans. Power Deliv., 30, 878 (2015)

20. X. Dou, J. Wang, Z. Wang et al., INT J ELEC POWER, 124, 106356 (2021) 